\title{
Structures of Small Tantalum Cluster Anions: Experiment and Theory
}

\author{
Manuel Kraft, ${ }^{a}$ Jesús R. Flores, ${ }^{\mathrm{b} *}$ Wim Klopper, ${ }^{\mathrm{a}, \mathrm{c}}$ Manfred M. Kappes ${ }^{\mathrm{a}, \mathrm{c}}$ and Detlef Schooss ${ }^{\mathrm{a} \#}$ \\ a) Karlsruhe Institute of Technology (KIT), Institute of Nanotechnology, \\ KIT Campus North, P.O. Box 3640, 76021 Karlsruhe, Germany \\ b) Departamento de Química Física, Facultade de Química, \\ Universidade de Vigo, 36310 Vigo, Pontevedra, Spain \\ c) Karlsruhe Institute of Technology (KIT), Institute of Physical Chemistry, \\ KIT Campus South, P.O. Box 6980, 76049 Karlsruhe, Germany
}

\section{Supplementary Information}

TABLE S1: Relative energy comparison for the most important isomers of the $\operatorname{Ta}_{n}{ }^{-}$clusters $(n=6-13)$. Structures are numbered as in Tables 1 to 8. The profile factors (TPSSh/def2-SVP) and the number of unpaired electrons (2C-BH-LYP/dhf-TZVP-2c) are also given.

\begin{tabular}{|c|c|c|c|c|c|c|c|c|c|c|}
\hline \multirow[b]{2}{*}{ Cluster } & & \multirow[b]{2}{*}{ Symmetry } & \multicolumn{7}{|c|}{$\Delta \mathrm{E}(\mathrm{eV})$} & \multirow[b]{2}{*}{$\begin{array}{c}R_{w} \\
\text { (TPSSh/ } \\
\text { def2- } \\
\text { SVP) }\end{array}$} \\
\hline & & & $\begin{array}{c}\text { Electronic } \\
\text { State }\end{array}$ & $\begin{array}{c}\text { TPSSh/ } \\
\text { def2- } \\
\text { SVP }\end{array}$ & $\begin{array}{l}\text { RPA@TPSSh/ } \\
\text { def2-SVP }\end{array}$ & $\begin{array}{c}\text { TPSSh/def2- } \\
\text { TZVPPD }\end{array}$ & $\begin{array}{l}\text { RPA@TPSSh/ } \\
\text { def2-TZVPPD }\end{array}$ & $\begin{array}{l}\text { 2C-BH-LYP/ } \\
\text { dhf-TZVP-2c }\end{array}$ & $\mathrm{N}_{\mathrm{s}}(2 \mathrm{C})$ & \\
\hline \multicolumn{11}{|l|}{$\mathrm{Ta}_{6}{ }^{-}$} \\
\hline & 1 & $\mathrm{C}_{1}$ & ${ }^{2} \mathrm{~A}$ & 0 & 0 & 0.10 & 0.01 & 0.24 & 2.82 & 0.060 \\
\hline & 2 & $D_{2 h}$ & ${ }^{4} A_{g}$ & 0.04 & 0.15 & 0 & 0 & 0 & 2.99 & 0.046 \\
\hline & 3 & $D_{2 h}$ & ${ }^{2} \mathrm{~B}_{3 \mathrm{~g}}$ & 0.06 & 0.08 & 0.14 & 0.01 & 0.04 & 2.93 & 0.054 \\
\hline \multicolumn{11}{|l|}{$\mathrm{Ta}_{7}{ }^{-}$} \\
\hline & 1 & $\mathrm{C}_{1}$ & ${ }^{1} \mathrm{~A}$ & 0 & 0 & 0 & 0 & 0.03 & 0.03 & 0.041 \\
\hline & 2 & $\mathrm{C}_{1}$ & ${ }^{3} \mathrm{~A}$ & 0.16 & 0.16 & 0.07 & 0.11 & 0 & 0.75 & 0.039 \\
\hline & 3 & $C_{3 v}$ & ${ }^{5} A_{1}$ & 1.21 & 1.37 & 1.15 & 1.31 & 1.06 & 1.96 & 0.042 \\
\hline & 4 & $\mathrm{C}_{\mathrm{s}}$ & ${ }^{3} \mathrm{~A} "$ & 1.29 & 1.33 & 1.28 & 1.30 & 1.17 & 2.46 & 0.045 \\
\hline & 5 & $\mathrm{C}_{1}$ & ${ }^{1} \mathrm{~A}$ & 1.94 & 1.94 & 1.83 & 1.95 & 1.60 & 0.58 & 0.048 \\
\hline \multicolumn{11}{|l|}{$\mathrm{Ta}_{8}^{-}$} \\
\hline & 1 & $C_{2 v}$ & ${ }^{2} \mathrm{~B}_{1}$ & 0 & 0 & 0 & 0 & 0 & 1.02 & 0.042 \\
\hline
\end{tabular}

\footnotetext{
* Corresponding author, e-mail: flores@uvigo.es

\# Corresponding author, e-mail: detlef.schooss@kit.edu
} 


$\begin{array}{llllllllll}2 & \mathrm{C}_{s} & { }^{4} \mathrm{~A}^{\prime} & 0.96 & 1.02 & 0.86 & 1.00 & 0.39 & 2.84 & 0.040 \\ 3 & \mathrm{C}_{3 \mathrm{v}} & { }^{2} \mathrm{~A}_{1} & 1.09 & 1.23 & 0.86 & 1.15 & 0.29 & 0.54 & 0.085 \\ 6 & \mathrm{D}_{2 \mathrm{~d}} & { }^{6} \mathrm{~B}_{2} & 1.35 & 1.43 & 1.18 & 1.35 & 0.47 & 2.77 & 0.17 \\ 7 & \mathrm{C}_{5} & { }^{4} \mathrm{~A}^{\prime} & 1.71 & 1.83 & 1.70 & 1.82 & 1.36 & 3.21 & 0.055 \\ 8 & \mathrm{C}_{2 \mathrm{v}} & { }^{4} \mathrm{~A}_{2} & 2.17 & 2.13 & 2.07 & 2.11 & 1.46 & 2.96 & 0.042\end{array}$

Tag ${ }^{-}$

$\begin{array}{lccccccccc}1 & \mathrm{C}_{2 \mathrm{v}} & { }^{3} \mathrm{~B}_{2} & 0 & 0 & 0 & 0 & 0 & 1.97 & 0.029 \\ 2 & \mathrm{C}_{1} & { }^{3} \mathrm{~A} & 0.16 & 0.27 & 0.21 & 0.29 & 0.32 & 0.22 & 0.10 \\ 3 & \mathrm{C}_{s} & { }^{5} \mathrm{~A} " & 0.90 & 1.09 & 0.99 & 1.20 & 0.89 & 3.79 & 0.048 \\ 4 & \mathrm{C}_{1} & { }^{1} \mathrm{~A} & 1.55 & 1.50 & 1.81 & 1.74 & 1.76 & 5.60 & 0.045\end{array}$

$\mathrm{Ta}_{10}{ }^{-}$

$\begin{array}{cccccccccc}1 & \mathrm{D}_{4 d} & { }^{2} \mathrm{~B}_{1} & 0 & 0 & 0 & 0 & 0.03 & 0.95 & 0.13 \\ 2 & \mathrm{C}_{3 \mathrm{v}} & { }^{4} \mathrm{~A}_{2} & 0.47 & 0.52 & 0.24 & 0.27 & 0 & 0.08 & 0.029 \\ 3 & \mathrm{C}_{s} & { }^{2} \mathrm{~A}^{\prime} & 1.72 & 1.55 & 1.67 & 1.52 & 1.59 & 0.87 & 0.046 \\ 4 & \mathrm{C}_{s} & { }^{2} \mathrm{~A}^{\prime} & 1.93 & 1.84 & 1.79 & 1.74 & 1.25 & 2.89 & 0.049\end{array}$

$\mathrm{Ta}_{11}{ }^{-}$

$\begin{array}{lccccccccc}1 & \mathrm{C}_{2 v} & { }^{3} \mathrm{~B}_{2} & 0 & 0 & 0 & 0 & 0 & 2.08 & 0.040 \\ 2 & \mathrm{C}_{s} & { }^{3} \mathrm{~A}^{\prime \prime} & 0.24 & 0.03 & 0.26 & 0.18 & 0.39 & 0.69 & 0.048 \\ 3 & \mathrm{C}_{s} & { }^{5} \mathrm{~A}^{\prime} & 0.31 & 0.28 & 0.24 & 0.29 & 0.12 & 4.12 & 0.030 \\ 4 & \mathrm{C}_{s} & { }^{3} \mathrm{~A}^{\prime \prime} & 0.51 & 0.43 & 0.57 & 0.60 & 0.61 & 1.71 & 0.074 \\ 5 & \mathrm{C}_{1} & { }^{3} \mathrm{~A} & 0.75 & 0.76 & 0.89 & 0.87 & 0.81 & 0.44 & 0.035 \\ 6 & \mathrm{C}_{1} & { }^{3} \mathrm{~A} & 1.46 & 1.29 & 1.50 & 1.47 & 1.18 & 3.34 & 0.041 \\ 7 & \mathrm{C}_{2} & { }^{5} \mathrm{~A} & 1.48 & 1.39 & 1.53 & 1.52 & 1.12 & 3.61 & 0.036\end{array}$

$\mathrm{Ta}_{12}{ }^{-}$

\begin{tabular}{|c|c|c|c|c|c|c|c|c|c|}
\hline 1 & $C_{s}$ & ${ }^{2} A "$ & 0 & 0.17 & 0.33 & 0.10 & 1.06 & 0.86 & 0.096 \\
\hline 2 & $T_{h}$ & ${ }^{4} \mathrm{~A}_{\mathrm{u}}$ & 0.09 & 0.62 & 0 & 0 & 0 & 1.61 & 0.12 \\
\hline 3 & $C_{s}$ & ${ }^{2} A^{\prime \prime}$ & 0.09 & 0.10 & 0.45 & 0.13 & 0.99 & 1.98 & 0.051 \\
\hline $3-b$ & $C_{s}$ & ${ }^{4} A "$ & 0.13 & 0.18 & 0.50 & 0.22 & 1.05 & 2.45 & 0.055 \\
\hline $3-c$ & $C_{1}$ & ${ }^{6} \mathrm{~A}$ & 0.30 & 0.50 & 0.60 & 0.47 & 0.94 & 3.44 & 0.054 \\
\hline 4 & $\mathrm{C}_{1}$ & ${ }^{2} \mathrm{~A}$ & 0.16 & 0 & 0.58 & 0.15 & 1.32 & 0.52 & 0.029 \\
\hline 5 & $C_{1}$ & ${ }^{4} \mathrm{~A}$ & 0.25 & 0.20 & 0.62 & 0.31 & 1.19 & 2.26 & 0.023 \\
\hline 6 & $C_{s}$ & ${ }^{2} A^{\prime}$ & 0.44 & 0.61 & 0.69 & 0.48 & 1.24 & 1.02 & 0.057 \\
\hline 7 & $C_{s}$ & ${ }^{2} A^{\prime \prime}$ & 0.47 & 0.87 & 0.85 & 0.72 & 1.35 & 0.81 & 0.071 \\
\hline 8 & $C_{2 v}$ & ${ }^{2} \mathrm{~A}_{1}$ & 0.56 & 0.65 & 0.87 & 0.67 & 1.45 & 1.19 & 0.11 \\
\hline 9 & $C_{2}$ & ${ }^{2} \mathrm{~A}$ & 0.79 & 1.05 & 1.06 & 0.75 & 1.54 & 0.93 & 0.030 \\
\hline 10 & $C_{s}$ & ${ }^{4} A^{\prime}$ & 0.82 & 1.08 & 1.06 & 0.88 & 1.37 & 2.98 & 0.052 \\
\hline 11 & $C_{i}$ & ${ }^{2} \mathrm{Ag}_{\mathrm{g}}$ & 1.25 & 1.22 & 1.72 & 1.23 & 2.22 & 0.99 & 0.17 \\
\hline
\end{tabular}




$\begin{array}{llllllllll}12 & \mathrm{C}_{1} & { }^{4} \mathrm{~A} & 1.28 & 1.32 & 1.59 & 1.37 & 1.96 & 4.29 & 0.032 \\ 13 & \mathrm{C}_{2} & { }^{2} \mathrm{~B} & 1.31 & 1.64 & 1.85 & 1.58 & 2.12 & 1.13 & 0.047\end{array}$

$\mathrm{Ta}_{13}{ }^{-}$

$\begin{array}{rccccccccc}1 & \mathrm{C}_{s} & { }^{1} \mathrm{~A}^{\prime} & 0 & 0.39 & 0.07 & 0.21 & 0.89 & 0.59 & 0.13 \\ 2 & \mathrm{C}_{2 v} & { }^{1} \mathrm{~A}_{1} & 0.08 & 0.12 & 0.17 & 0.28 & 0.65 & 4.67 & 0.039 \\ 3 & \mathrm{C}_{s} & { }^{3} \mathrm{~A}^{\prime \prime} & 0.13 & 0 & 0 & 0 & 0 & 0.90 & 0.017 \\ 4 & \mathrm{C}_{2} & { }^{1} \mathrm{~A} & 0.21 & 0.32 & 0.46 & 0.46 & 1.25 & 0.78 & 0.12 \\ 5 & \mathrm{C}_{s} & { }^{3} \mathrm{~A}^{\prime} & 0.23 & 0.56 & 0.21 & 0.38 & 0.74 & 2.00 & 0.091\end{array}$


TABLE S2: Dispersion corrections calculated by the D3, D3-BJ and D4 methods relative to the lowest-lying isomer obtained at the TPSSh/def2-SVP level, and corrected TPSSh/def2-SVP relative energies. Standard options and the Axilrod-Teller-Muto three-body dispersion term are used.

\begin{tabular}{|c|c|c|c|c|c|c|c|c|c|c|}
\hline \multirow[b]{2}{*}{ Cluster } & \multirow[b]{2}{*}{ Isomer } & \multirow[b]{2}{*}{ Symmetry } & \multirow[b]{2}{*}{$\begin{array}{c}\text { Electronic } \\
\text { State }\end{array}$} & \multicolumn{5}{|c|}{$\Delta \mathrm{E}(\mathrm{eV})$} & \multirow[b]{2}{*}{$\begin{array}{c}\text { TPSSh/def2-SVP } \\
\text { + D3-BJ }\end{array}$} & \multirow[b]{2}{*}{$\begin{array}{c}\text { TPSSh/d } \\
\text { ef2-SVP } \\
+ \text { D4 }\end{array}$} \\
\hline & & & & $\mathrm{D} 3^{(\mathrm{a})}$ & D3-BJ(b) & $\mathrm{D} 4^{(c)}$ & $\begin{array}{l}\text { TPSSh/def2 } \\
\text {-SVP }\end{array}$ & $\begin{array}{c}\text { TPSSh/def2- } \\
\text { SVP + D3 }\end{array}$ & & \\
\hline \multicolumn{11}{|l|}{$\mathrm{Ta}_{6}{ }^{-}$} \\
\hline & 1 & $\mathrm{C}_{1}$ & ${ }^{2} \mathrm{~A}$ & 0.000 & 0.000 & 0.000 & 0.00 & 0.00 & 0.00 & 0.00 \\
\hline & 2 & $D_{2 h}$ & ${ }^{4} A_{g}$ & -0.002 & 0.000 & 0.001 & 0.04 & 0.03 & 0.04 & 0.04 \\
\hline & 3 & $D_{2 h}$ & ${ }^{2} \mathrm{~B}_{3 \mathrm{~g}}$ & -0.007 & 0.001 & 0.001 & 0.06 & 0.05 & 0.06 & 0.06 \\
\hline \multicolumn{11}{|l|}{$\mathrm{Ta}_{7}^{-}$} \\
\hline & 1 & $\mathrm{C}_{1}$ & ${ }^{1} \mathrm{~A}$ & 0.000 & 0.000 & 0.000 & 0.00 & 0.00 & 0.00 & 0.00 \\
\hline & 2 & $\mathrm{C}_{1}$ & ${ }^{3} \mathrm{~A}$ & -0.001 & 0.000 & 0.000 & 0.16 & 0.16 & 0.16 & 0.16 \\
\hline & 3 & $C_{3 v}$ & ${ }^{5} A_{1}$ & -0.010 & 0.001 & -0.039 & 1.21 & 1.20 & 1.21 & 1.17 \\
\hline & 4 & $\mathrm{C}_{\mathrm{s}}$ & ${ }^{3} \mathrm{~A}^{\prime \prime}$ & -0.006 & 0.001 & -0.039 & 1.29 & 1.29 & 1.29 & 1.25 \\
\hline & 5 & $\mathrm{C}_{1}$ & ${ }^{1} \mathrm{~A}$ & -0.038 & 0.012 & -0.074 & 1.94 & 1.90 & 1.95 & 1.86 \\
\hline \multicolumn{11}{|l|}{$\mathrm{Ta}_{8}^{-}$} \\
\hline & 1 & $C_{2 v}$ & ${ }^{2} \mathrm{~B}_{1}$ & 0.000 & 0.000 & 0.000 & 0.00 & 0.00 & 0.00 & 0.00 \\
\hline & 2 & $\mathrm{C}_{s}$ & ${ }^{4} A^{\prime}$ & -0.031 & 0.015 & -0.036 & 0.96 & 0.93 & 0.97 & 0.92 \\
\hline & 3 & $C_{3 v}$ & ${ }^{2} \mathrm{~A}_{1}$ & -0.042 & 0.018 & 0.008 & 1.09 & 1.05 & 1.11 & 1.10 \\
\hline & 6 & $D_{2 d}$ & ${ }^{6} \mathrm{~B}_{2}$ & 0.008 & 0.033 & 0.016 & 1.35 & 1.35 & 1.38 & 1.36 \\
\hline & 7 & $\mathrm{C}_{s}$ & ${ }^{4} A^{\prime}$ & -0.016 & 0.021 & -0.077 & 1.71 & 1.69 & 1.73 & 1.63 \\
\hline & 8 & $C_{2 v}$ & ${ }^{4} \mathrm{~A}_{2}$ & -0.060 & 0.014 & -0.085 & 2.17 & 2.11 & 2.19 & 2.09 \\
\hline \multicolumn{11}{|l|}{$\mathrm{Ta}_{9}{ }^{-}$} \\
\hline & 1 & $C_{2 v}$ & ${ }^{3} \mathrm{~B}_{2}$ & 0.000 & 0.000 & 0.000 & 0.00 & 0.00 & 0.00 & 0.00 \\
\hline & 2 & $\mathrm{C}_{1}$ & ${ }^{3} \mathrm{~A}$ & 0.013 & -0.020 & -0.009 & 0.16 & 0.17 & 0.14 & 0.15 \\
\hline & 3 & $C_{s}$ & ${ }^{5} A^{\prime \prime}$ & 0.015 & 0.007 & 0.003 & 0.90 & 0.92 & 0.91 & 0.90 \\
\hline & 4 & $C_{1}$ & ${ }^{1} \mathrm{~A}$ & 0.017 & 0.020 & -0.085 & 1.55 & 1.57 & 1.58 & 1.47 \\
\hline \multicolumn{11}{|l|}{$\mathrm{Ta}_{10}{ }^{-}$} \\
\hline & 1 & $D_{4 d}$ & ${ }^{2} \mathrm{~B}_{1}$ & 0.000 & 0.000 & 0.000 & 0.00 & 0.00 & 0.00 & 0.00 \\
\hline & 2 & $C_{3 v}$ & ${ }^{4} \mathrm{~A}_{2}$ & 0.002 & 0.016 & 0.007 & 0.47 & 0.47 & 0.49 & 0.48 \\
\hline & 3 & $\mathrm{C}_{\mathrm{s}}$ & ${ }^{2} A^{\prime}$ & 0.048 & 0.059 & -0.021 & 1.72 & 1.77 & 1.78 & 1.70 \\
\hline & 4 & $\mathrm{C}_{\mathrm{s}}$ & ${ }^{2} A^{\prime}$ & 0.043 & 0.073 & 0.033 & 1.93 & 1.97 & 2.00 & 1.97 \\
\hline \multicolumn{11}{|l|}{$\mathrm{Ta}_{11}{ }^{-}$} \\
\hline & 1 & $C_{2 v}$ & ${ }^{3} \mathrm{~B}_{2}$ & 0.000 & 0.000 & 0.000 & 0.00 & 0.00 & 0.00 & 0.00 \\
\hline & 2 & $C_{s}$ & ${ }^{3} A^{\prime \prime}$ & 0.077 & 0.033 & 0.015 & 0.24 & 0.32 & 0.28 & 0.26 \\
\hline & 3 & $C_{s}$ & ${ }^{5} A^{\prime}$ & 0.036 & 0.021 & 0.009 & 0.31 & 0.35 & 0.33 & 0.32 \\
\hline
\end{tabular}




$\begin{array}{llllllllll}4 & \mathrm{C}_{5} & { }^{3} \mathrm{~A}^{\prime \prime} & 0.074 & 0.041 & 0.019 & 0.51 & 0.58 & 0.55 & 0.52 \\ 5 & \mathrm{C}_{1} & { }^{3} \mathrm{~A} & 0.026 & 0.008 & 0.003 & 0.75 & 0.78 & 0.76 & 0.75 \\ 6 & \mathrm{C}_{1} & { }^{3} \mathrm{~A} & 0.056 & 0.046 & 0.021 & 1.46 & 1.51 & 1.50 & 1.48 \\ 7 & \mathrm{C}_{2} & { }^{5} \mathrm{~A} & 0.047 & 0.042 & 0.019 & 1.48 & 1.53 & 1.52 & 1.50\end{array}$

$\mathrm{Ta}_{12}$

$\begin{array}{cccccccccc}1 & \mathrm{C}_{\mathrm{s}} & { }^{2} \mathrm{~A}^{\prime \prime} & 0.000 & 0.000 & 0.000 & 0.00 & 0.00 & 0.00 & 0.00 \\ 2 & \mathrm{~T}_{\mathrm{h}} & { }^{4} \mathrm{~A}_{\mathrm{u}} & -0.262 & -0.059 & -0.027 & 0.09 & -0.18 & 0.03 & 0.06 \\ 3 & \mathrm{C}_{\mathrm{s}} & { }^{2} \mathrm{~A}^{\prime \prime} & -0.024 & -0.028 & -0.005 & 0.09 & 0.06 & 0.08 & 0.08 \\ 3-\mathrm{b} & \mathrm{C}_{\mathrm{s}} & { }^{4} \mathrm{~A}^{\prime \prime} & -0.016 & -0.007 & -0.003 & 0.13 & 0.11 & 0.12 & 0.13 \\ 3-\mathrm{c} & \mathrm{C}_{1} & { }^{6} \mathrm{~A} & -0.018 & -0.009 & -0.004 & 0.30 & 0.29 & 0.30 & 0.30 \\ 4 & \mathrm{C}_{1} & { }^{2} \mathrm{~A} & -0.044 & -0.028 & -0.012 & 0.16 & 0.12 & 0.14 & 0.15 \\ 5 & \mathrm{C}_{1} & { }^{4} \mathrm{~A} & -0.047 & -0.028 & -0.012 & 0.25 & 0.21 & 0.23 & 0.24 \\ 6 & \mathrm{C}_{\mathrm{s}} & { }^{2} \mathrm{~A}^{\prime} & -0.028 & 0.011 & 0.005 & 0.44 & 0.41 & 0.45 & 0.44 \\ 7 & \mathrm{C}_{\mathrm{s}} & { }^{2} \mathrm{~A}^{\prime \prime} & -0.154 & -0.039 & -0.018 & 0.47 & 0.32 & 0.43 & 0.45 \\ 8 & \mathrm{C}_{2 v} & { }^{2} \mathrm{~A}_{1} & 0.018 & 0.042 & 0.019 & 0.56 & 0.57 & 0.60 & 0.57 \\ 9 & \mathrm{C}_{2} & { }^{2} \mathrm{~A} & -0.057 & -0.010 & -0.005 & 0.79 & 0.73 & 0.78 & 0.79 \\ 10 & \mathrm{C}_{5} & { }^{4} \mathrm{~A}^{\prime} & 0.061 & 0.075 & -0.005 & 0.82 & 0.88 & 0.89 & 0.81 \\ 11 & \mathrm{C}_{\mathrm{i}} & { }^{2} \mathrm{~A}_{\mathrm{g}} & 0.121 & 0.051 & 0.022 & 1.25 & 1.37 & 1.30 & 1.28 \\ 12 & \mathrm{C}_{1} & { }^{4} \mathrm{~A} & 0.010 & 0.051 & -0.028 & 1.28 & 1.29 & 1.33 & 1.25 \\ 13 & \mathrm{C}_{2} & { }^{2} \mathrm{~B} & -0.095 & -0.023 & -0.010 & 1.31 & 1.22 & 1.29 & 1.30\end{array}$

$\mathrm{Ta}_{13}{ }^{-}$

$\begin{array}{llllllllll}1 & \mathrm{C}_{s} & { }^{1} \mathrm{~A}^{\prime} & 0.000 & 0.000 & 0.000 & 0.00 & 0.00 & 0.00 & 0.00 \\ 2 & \mathrm{C}_{2 v} & { }^{1} \mathrm{~A}_{1} & -0.046 & -0.078 & -0.035 & 0.08 & 0.03 & 0.00 & 0.05 \\ 3 & \mathrm{C}_{s} & { }^{3} \mathrm{~A}^{\prime \prime} & -0.080 & -0.086 & -0.039 & 0.13 & 0.05 & 0.04 & 0.09 \\ 4 & \mathrm{C}_{2} & { }^{1} \mathrm{~A} & 0.007 & -0.018 & -0.008 & 0.21 & 0.21 & 0.19 & 0.20 \\ 5 & \mathrm{C}_{s} & { }^{3} \mathrm{~A}^{\prime} & -0.033 & -0.019 & -0.008 & 0.23 & 0.20 & 0.22 & 0.23\end{array}$

(a) Grimme, S., Antony, J., Ehrlich, S., Krieg, H., J. Chem. Phys, 132 (2010), 154104.

(b) Grimme, S., Ehrlich, S., Goerigk, L., J. Comput. Chem. 32 (2011), 1456-1465

(c) Caldeweyher, E., Bannwarth, C., Grimme, S., J. Chem. Phys., 147 (2017), 034112; Caldeweyher, E., Ehlert, S., Hansen,

A., Neugebauer, H., Spicher, S., Bannwarth, C., Grimme, S., J. Chem. Phys., 150 (2019), 154122. 
TABLE S3: Relative internal energy, chemical potential and relative chemical potential computed by the TPSSh/def2-SVP method using harmonic frequencies at T=95 $\mathrm{K}$ and $\mathrm{p}=0.1 \mathrm{MPa}$.

\begin{tabular}{ccccccc}
\hline Cluster & Isomer & Symmetry & $\begin{array}{c}\text { Electronic } \\
\text { State }\end{array}$ & $\begin{array}{c}\Delta \mathrm{U}(\mathrm{eV}) \\
\text { TPSSh/def2-SVP }\end{array}$ & $\begin{array}{c}\mu(\mathrm{eV}) \\
\text { TPSSh/def2-SVP }\end{array}$ & $\begin{array}{c}\Delta \mu(\mathrm{eV}) \\
\text { TPSSh/def2-SVP }\end{array}$ \\
\hline $\mathrm{Ta}_{6}{ }^{-}$ & & & & & -0.18 & 0.00 \\
& 1 & $\mathrm{C}_{1}$ & ${ }^{2} \mathrm{~A}$ & 0.00 & -0.15 & 0.03 \\
& 2 & $\mathrm{D}_{2 \mathrm{~h}}$ & ${ }^{4} \mathrm{~A}_{\mathrm{g}}$ & 0.03 & -0.12 & 0.06 \\
& & $\mathrm{D}_{2 \mathrm{~h}}$ & ${ }^{2} \mathrm{~B}_{3 \mathrm{~g}}$ & 0.06 & 0.06
\end{tabular}

$\mathrm{Ta}^{-}$

$\begin{array}{llllll}1 & \mathrm{C}_{1} & { }^{1} \mathrm{~A} & 0.00 & -0.17 & 0.00 \\ 2 & \mathrm{C}_{1} & { }^{3} \mathrm{~A} & 0.16 & -0.01 & 0.15 \\ 3 & \mathrm{C}_{3 \mathrm{v}} & { }^{5} \mathrm{~A}_{1} & 1.20 & 1.04 & 1.20 \\ 4 & \mathrm{C}_{5} & { }^{3} \mathrm{~A} " & 1.28 & 1.10 & 1.27 \\ 5 & \mathrm{C}_{1} & { }^{1} \mathrm{~A} & 1.93 & 1.76 & 1.92\end{array}$

$\mathrm{Ta}_{8}{ }^{-}$

$\begin{array}{llllll}1 & \mathrm{C}_{2 \mathrm{v}} & { }^{2} \mathrm{~B}_{1} & 0.00 & -0.13 & 0.00 \\ 2 & \mathrm{C}_{\mathrm{s}} & { }^{4} \mathrm{~A}^{\prime} & 0.95 & 0.80 & 0.93 \\ 3 & \mathrm{C}_{3 \mathrm{v}} & { }^{2} \mathrm{~A}_{1} & 1.09 & 0.96 & 1.09 \\ 6 & \mathrm{D}_{2 \mathrm{~d}} & { }^{6} \mathrm{~B}_{2} & 1.34 & 1.19 & 1.31 \\ 7 & \mathrm{C}_{\mathrm{s}} & { }^{4} \mathrm{~A}^{\prime} & 1.70 & 1.53 & 1.66 \\ 8 & \mathrm{C}_{2 \mathrm{v}} & { }^{4} \mathrm{~A}_{2} & 2.17 & 2.03 & 2.15\end{array}$

$\mathrm{Ta}_{9}{ }^{-}$

$\begin{array}{llllll}1 & \mathrm{C}_{2 \mathrm{v}} & { }^{3} \mathrm{~B}_{2} & 0.00 & -0.14 & 0.00 \\ 2 & \mathrm{C}_{1} & { }^{3} \mathrm{~A} & 0.16 & 0.02 & 0.16 \\ 3 & \mathrm{C}_{5} & { }^{5} \mathrm{~A} " & 0.90 & 0.76 & 0.91 \\ 4 & \mathrm{C}_{1} & { }^{1} \mathrm{~A} & 1.55 & 1.41 & 1.55\end{array}$

$\mathrm{Ta}_{10}{ }^{-}$

$\begin{array}{llllll}1 & \mathrm{D}_{4 \mathrm{~d}} & { }^{2} \mathrm{~B}_{1} & 0.00 & -0.07 & 0.00 \\ 2 & \mathrm{C}_{3 \mathrm{v}} & { }^{4} \mathrm{~A}_{2} & 0.47 & 0.37 & 0.44 \\ 3 & \mathrm{C}_{s} & { }^{2} \mathrm{~A}^{\prime} & 1.71 & 1.60 & 1.67 \\ 4 & \mathrm{C}_{s} & { }^{2} \mathrm{~A}^{\prime} & 1.92 & 1.81 & 1.88\end{array}$

$\mathrm{Ta}_{11}$

$\begin{array}{llllll}1 & \mathrm{C}_{2 v} & { }^{3} \mathrm{~B}_{2} & 0.00 & -0.10 & 0.00 \\ 2 & \mathrm{C}_{s} & { }^{3} \mathrm{~A}^{\prime \prime} & 0.24 & 0.12 & 0.23 \\ 3 & \mathrm{C}_{s} & { }^{5} \mathrm{~A}^{\prime} & 0.31 & 0.19 & 0.29 \\ 4 & \mathrm{C}_{s} & { }^{3} \mathrm{~A}^{\prime \prime} & 0.51 & 0.41 & 0.51 \\ 5 & \mathrm{C}_{1} & { }^{3} \mathrm{~A} & 0.75 & 0.64 & 0.74\end{array}$




$\begin{array}{llllll}6 & \mathrm{C}_{1} & { }^{3} \mathrm{~A} & 1.45 & 1.33 & 1.43 \\ 7 & \mathrm{C}_{2} & { }^{5} \mathrm{~A} & 1.47 & 1.35 & 1.46\end{array}$

$\mathrm{Ta}_{12}$

$\begin{array}{cccccc}1 & \mathrm{C}_{s} & { }^{2} \mathrm{~A}^{\prime \prime} & 0.00 & -0.08 & 0.00 \\ 2 & \mathrm{~T}_{\mathrm{h}} & { }^{4} \mathrm{~A}_{\mathrm{u}} & 0.08 & 0.02 & 0.09 \\ 3 & \mathrm{C}_{s} & { }^{2} \mathrm{~A}^{\prime \prime} & 0.08 & -0.01 & 0.06 \\ 3-\mathrm{b} & \mathrm{C}_{s} & { }^{4} \mathrm{~A}^{\prime \prime} & 0.12 & 0.02 & 0.10 \\ 3-\mathrm{c} & \mathrm{C}_{1} & { }^{6} \mathrm{~A} & 0.29 & 0.19 & 0.26 \\ 4 & \mathrm{C}_{1} & { }^{2} \mathrm{~A} & 0.16 & 0.05 & 0.13 \\ 5 & \mathrm{C}_{1} & { }^{4} \mathrm{~A} & 0.24 & 0.12 & 0.20 \\ 6 & \mathrm{C}_{s} & { }^{2} \mathrm{~A}^{\prime} & 0.44 & 0.35 & 0.43 \\ 7 & \mathrm{C}_{s} & { }^{2} \mathrm{~A}^{\prime \prime} & 0.47 & 0.40 & 0.47 \\ 8 & \mathrm{C}_{2 v} & { }^{2} \mathrm{~A}_{1} & 0.56 & 0.48 & 0.56 \\ 9 & \mathrm{C}_{2} & { }^{2} \mathrm{~A} & 0.79 & 0.71 & 0.78 \\ 10 & \mathrm{C}_{s} & { }^{4} \mathrm{~A}^{\prime} & 0.81 & 0.71 & 0.79 \\ 11 & \mathrm{C}_{\mathrm{i}} & { }^{2} \mathrm{~A}_{\mathrm{g}} & 1.25 & 1.16 & 1.24 \\ 12 & \mathrm{C}_{1} & { }^{4} \mathrm{~A} & 1.27 & 1.16 & 1.24 \\ 13 & \mathrm{C}_{2} & { }^{2} \mathrm{~B} & 1.30 & 1.23 & 1.31\end{array}$

$\mathrm{Ta}_{13}$

$\begin{array}{llllll}1 & \mathrm{C}_{s} & { }^{1} \mathrm{~A}^{\prime} & 0.00 & -0.05 & 0.00 \\ 2 & \mathrm{C}_{2 \mathrm{v}} & { }^{1} \mathrm{~A}_{1} & 0.07 & -0.01 & 0.04 \\ 3 & \mathrm{C}_{5} & { }^{3} \mathrm{~A}^{\prime \prime} & 0.12 & 0.04 & 0.09 \\ 4 & \mathrm{C}_{2} & { }^{1} \mathrm{~A} & 0.20 & 0.13 & 0.18 \\ 5 & \mathrm{C}_{s} & { }^{3} \mathrm{~A}^{\prime} & 0.23 & 0.16 & 0.21\end{array}$


TABLE S4: RPA and TPSSh basis set convergence test by single-point computations on TPSSh/def2-SVP optimized geometries.

\begin{tabular}{|c|c|c|c|c|c|c|c|c|c|}
\hline \multirow[b]{2}{*}{ Cluster } & & \multirow[b]{2}{*}{ Symmetry } & \multicolumn{6}{|c|}{$\Delta \mathrm{E}(\mathrm{eV})$} & \multirow[b]{2}{*}{$\begin{array}{l}\text { RPA@TPSSh/ } \\
\text { def2-QZVPPD }\end{array}$} \\
\hline & & & $\begin{array}{c}\text { Electronic } \\
\text { State }\end{array}$ & $\begin{array}{l}\text { TPSSh/ } \\
\text { def2-SVP }\end{array}$ & $\begin{array}{l}\text { RPA@TPSSh/ } \\
\text { def2-SVP }\end{array}$ & $\begin{array}{c}\text { TPSSh/def2- } \\
\text { TZVPPD }\end{array}$ & $\begin{array}{l}\text { RPA@TPSSh/ } \\
\text { def2-TZVPPD }\end{array}$ & $\begin{array}{l}\text { TPSSh/def2- } \\
\text { QZVPPD(a) }\end{array}$ & \\
\hline \multicolumn{10}{|l|}{$\mathrm{Ta}_{6}{ }^{-}$} \\
\hline & 1 & $\mathrm{C}_{1}$ & ${ }^{2} \mathrm{~A}$ & 0 & 0 & 0.10 & 0.01 & 0.09 & 0 \\
\hline & 2 & $D_{2 h}$ & ${ }^{4} \mathrm{Ag}$ & 0.04 & 0.15 & 0 & 0 & 0 & 0.01 \\
\hline & 3 & $D_{2 h}$ & ${ }^{2} B_{3 g}$ & 0.06 & 0.08 & 0.14 & 0.01 & 0.14 & 0.01 \\
\hline
\end{tabular}

$\mathrm{Ta}_{7}$

$\begin{array}{ccccccccc}1 & \mathrm{C}_{1} & { }^{1} \mathrm{~A} & 0 & 0 & 0 & 0 & 0 & 0 \\ 3 & \mathrm{C}_{3 v} & { }^{5} \mathrm{~A}_{1} & 1.21 & 1.37 & 1.15 & 1.31 & 1.15 & 1.30\end{array}$

$\mathrm{Ta}^{-}$

$\begin{array}{ccccccccc}1 & \mathrm{C}_{2 v} & { }^{2} \mathrm{~B}_{1} & 0 & 0 & 0 & 0 & 0 & 0 \\ 2 & \mathrm{C}_{5} & { }^{4} \mathrm{~A}^{\prime} & 0.96 & 1.02 & 0.86 & 1.00 & 0.85 & 0.97 \\ 3 & \mathrm{C}_{3 v} & { }^{2} \mathrm{~A}_{1} & 1.09 & 1.23 & 0.86 & 1.15 & 0.86 & 1.04\end{array}$

$\mathrm{Tag}^{-}$

$\begin{array}{ccccccccc}1 & \mathrm{C}_{2 v} & { }^{3} \mathrm{~B}_{2} & 0 & 0 & 0 & 0 & 0 & 0 \\ 2 & \mathrm{C}_{1} & { }^{3} \mathrm{~A} & 0.16 & 0.27 & 0.21 & 0.29 & 0.21 & 0.27\end{array}$

$\mathrm{Ta}_{10}$

$\begin{array}{ccccccccc}1 & \mathrm{D}_{4 \mathrm{~d}} & { }^{2} \mathrm{~B}_{1} & 0 & 0 & 0 & 0 & 0 & 0 \\ 2 & \mathrm{C}_{3 \mathrm{v}} & { }^{4} \mathrm{~A}_{2} & 0.47 & 0.52 & 0.24 & 0.27 & 0.24 & 0.27\end{array}$

$\mathrm{Ta}_{11}{ }^{-}$

$\begin{array}{ccccccccc}1 & \mathrm{C}_{2 v} & { }^{3} \mathrm{~B}_{2} & 0 & 0 & 0 & 0 & 0 & 0 \\ 2 & \mathrm{C}_{s} & { }^{3} \mathrm{~A}^{\prime \prime} & 0.24 & 0.03 & 0.26 & 0.18 & 0.26 & 0.14 \\ 3 & \mathrm{C}_{s} & { }^{5} \mathrm{~A}^{\prime} & 0.31 & 0.28 & 0.24 & 0.29 & 0.24 & 0.27\end{array}$

$\mathrm{Ta}_{12}{ }^{-}$

$\begin{array}{ccccccccc}1 & \mathrm{C}_{\mathrm{s}} & { }^{2} \mathrm{~A}^{\prime \prime} & 0 & 0.17 & 0.33 & 0.10 & 0.32 & 0.03 \\ 2 & \mathrm{~T}_{\mathrm{h}} & { }^{4} \mathrm{~A}_{\mathrm{u}} & 0.09 & 0.62 & 0 & 0 & 0 & 0 \\ 3 & \mathrm{C}_{\mathrm{s}} & { }^{2} \mathrm{~A}^{\prime \prime} & 0.09 & 0.10 & 0.45 & 0.13 & 0.43 & 0.07 \\ 3-\mathrm{b} & \mathrm{C}_{\mathrm{s}} & { }^{4} \mathrm{~A}^{\prime \prime} & 0.13 & 0.18 & 0.50 & 0.22 & 0.49 & 0.15 \\ 3-\mathrm{C} & \mathrm{C}_{1} & { }^{6} \mathrm{~A} & 0.30 & 0.50 & 0.60 & 0.47 & 0.59 & 0.40 \\ 4 & \mathrm{C}_{1} & { }^{2} \mathrm{~A} & 0.16 & 0 & 0.58 & 0.15 & 0.57 & 0.05 \\ 5 & \mathrm{C}_{1} & { }^{4} \mathrm{~A} & 0.25 & 0.20 & 0.62 & 0.31 & 0.61 & 0.20 \\ 6 & \mathrm{C}_{s} & { }^{2} \mathrm{~A}^{\prime} & 0.44 & 0.61 & 0.69 & 0.48 & 0.68 & 0.37 \\ 9 & \mathrm{C}_{2} & { }^{2} \mathrm{~A} & 0.79 & 1.05 & 1.06 & 0.75 & 1.05 & 0.69 \\ 10 & \mathrm{C}_{s} & { }^{4} \mathrm{~A}^{\prime} & 0.82 & 1.08 & 1.06 & 0.88 & 1.04 & 0.78\end{array}$


$\mathrm{Ta}_{13}{ }^{-}$

$\begin{array}{ccccccccc}1 & \mathrm{C}_{s} & { }^{1} \mathrm{~A}^{\prime} & 0 & 0.39 & 0.07 & 0.21 & 0.09 & 0.21 \\ 2 & \mathrm{C}_{2 v} & { }^{1} \mathrm{~A}_{1} & 0.08 & 0.12 & 0.17 & 0.28 & 0.23 & 0.26 \\ 3 & \mathrm{C}_{s} & { }^{3} \mathrm{~A}^{\prime \prime} & 0.13 & 0 & 0 & 0 & 0 & 0\end{array}$

(a) QZVPPD basis set: Rappoport, D., Furche, F.; J. Chem. Phys. 133, 134105 (2010); Hellweg, A., Rappoport, D. unpublished (cbas); Weigend, F.; Phys. Chem. Chem. Phys. 8, 1057 (2006) (jbas) . 
Table S5: Density functional dependence of selected motifs of the Ta12 cluster. Relative energies are defined with respect to isomer 2 (symmetric icosahedral cage).

\begin{tabular}{ccccc}
\hline & \multicolumn{4}{c}{$\Delta \mathrm{E}(\mathrm{eV})$} \\
\cline { 2 - 4 } & $\begin{array}{c}\mathbf{3}(\mathrm{pbp} / \text { inc. Ico } \\
\left.\left(\mathrm{C}_{\mathrm{s}}\right)\right)\end{array}$ & $\mathbf{2}$ (Ico cage $\left.\left(\mathrm{T}_{\mathrm{h}}\right)\right)$ & $\mathbf{1}\left(\mathrm{hbp}\left(\mathrm{C}_{\mathrm{s}}\right)\right)$ & $\mathbf{9}\left(\right.$ Ico-cage $\left.\left(\mathrm{C}_{2}\right)\right)$ \\
\cline { 2 - 5 } BH-LYP/def2-SVP & 0.44 & 0.00 & 0.35 & 1.00 \\
PBE/def2-SVP(a) & 0.39 & 0.00 & 0.27 & 0.95 \\
PBE0/def2-SVP(b) & 0.25 & 0.00 & 0.11 & 0.80 \\
PBEH-3C/def2-SVP(c) & 0.40 & 0.00 & 0.26 & 0.98 \\
TPSS/def2-SVP & -0.07 & 0.00 & -0.14 & 0.66 \\
TPSSh/def2-SVP & 0.00 & 0.00 & -0.09 & 0.70 \\
Lh14t-calPBE/def2- & 0.28 & 0.00 & -0.04 & 0.59 \\
SVP(d) & & & &
\end{tabular}
(a) Perdew, J.P., Burke, K., Ernzerhof, M., Phys. Rev. Lett. 77 (1996), 3865
(b) Perdew, J.P., Ernzerhof, M., Burke, K., J. Chem. Phys. 105 (1996), 9982
(c) Grimme, S., Brandenburg, J.G., Bannwarth, C., Hansen, A., J. Chem. Phys. 143 (2015), 054107. Includes geometrical counterpoise corrections and D3 dispersion corrections with BJ damping and three-body term (the relative energies without dispersion correction are the same up to $<0.01 \mathrm{eV}$ ).
(d) Arbuznikov, A.V., Kaupp M., J. Chem. Phys. 141 (2014), 204101; the reference grid has been employed.

Table S6: Basis set dependence of the 3-2 energy gap of $\mathrm{Ta}_{12}{ }^{-}(\mathrm{eV})$ computed with the BH-LYP and TPSSh density functionals.

\begin{tabular}{cccccc}
\hline $\begin{array}{c}\text { TPSSh/def2- } \\
\text { SVP }\end{array}$ & $\begin{array}{c}\text { TPSSh/def2- } \\
\text { QZVPPD }\end{array}$ & $\begin{array}{c}\text { TPSSh/dhf- } \\
\text { TZVP(a) }\end{array}$ & $\begin{array}{c}\text { BH-LYP/def2- } \\
\text { SVP }\end{array}$ & $\begin{array}{c}\text { BH-LYP/def2- } \\
\text { QZVPPD }\end{array}$ & $\begin{array}{c}\text { BH-LYP/dhf- } \\
\text { TZVP }\end{array}$ \\
\hline 0.00 & 0.43 & 0.39 & 0.44 & 1.00 & 0.91 \\
\hline
\end{tabular}

(a) Weigend, F., Baldes, A.; J. Chem. Phys. 133, 174102 (2010); Figgen, D., Peterson, K.A. , Dolg, M., Stoll, H.; J. Chem. Phys. 130, 164108 (2009); Weigend, F.; Phys. Chem. Chem. Phys. 8, 1057 (2006).

Table S7: DF dependence of selected motifs of the $\mathrm{Ta}_{10}{ }^{-}$cluster.

\begin{tabular}{ccc}
\hline & \multicolumn{2}{c}{$\Delta \mathrm{E}(\mathrm{eV})$} \\
\cline { 2 - 3 } & \multicolumn{1}{c}{$\mathbf{1}$ (bi-capped sap, $\mathrm{D}_{4 \mathrm{~d}}$ ) } & $\mathbf{2}$ (tri-capped pbp, $\mathrm{C}_{3 \mathrm{v}}$ ) \\
\cline { 2 - 3 } BH-LYP/def2-SVP & 0.00 & 0.35 \\
PBE/def2-SVP & 0.00 & 0.63 \\
PBE0/def2-SVP & 0.00 & 0.48 \\
PBEH-3C/def2-SVP & 0.00 & 0.42 \\
TPSS/def2-SVP & 0.00 & 0.52 \\
TPSSh/def2-SVP & 0.00 & 0.47 \\
Lh14t-calPBE/def2-SVP(a) & 0.00 & 0.71 \\
\hline
\end{tabular}

(a) The reference grid is used. 
Table S8: Basis set dependence of the 2-1 energy gap of $\mathrm{Ta}_{10}^{-}(\mathrm{eV})$ computed with the BH-LYP and TPSSh density functionals.

\begin{tabular}{cccccc}
\hline $\begin{array}{c}\text { TPSSh/def2- } \\
\text { SVP }\end{array}$ & $\begin{array}{c}\text { TPSSh/def2- } \\
\text { QZVPPD }\end{array}$ & $\begin{array}{c}\text { TPSSh/dhf- } \\
\text { TZVP(a) }\end{array}$ & BH-LYP/def2-SVP & $\begin{array}{c}\text { BH-LYP/def2- } \\
\text { QZVPPD }\end{array}$ & BH-LYP/dhf-TZVP \\
\hline 0.47 & 0.24 & 0.24 & 0.35 & 0.14 & 0.14 \\
\hline
\end{tabular}

Table S9: Detailed analysis of the electronic species of the symmetric icosahedral structure of $\mathrm{Ta}_{12}{ }^{-}{ }^{\text {(a) }}$

\begin{tabular}{|c|c|c|c|c|c|c|c|c|}
\hline \multirow[b]{2}{*}{ Symmetry } & \multirow[b]{2}{*}{$\begin{array}{c}\text { Electronic } \\
\text { State }\end{array}$} & \multicolumn{7}{|c|}{$\Delta \mathrm{E}(\mathrm{eV})$} \\
\hline & & $\begin{array}{l}\text { TPSSh/ } \\
\text { def2-SVP }\end{array}$ & $\begin{array}{l}\text { RPA@TPSSh/ } \\
\text { def2-SVP }\end{array}$ & $\begin{array}{l}\text { TPSSh/def2- } \\
\text { TZVPPD }\end{array}$ & $\begin{array}{l}\text { RPA@TPSSh/ } \\
\text { def2-TZVPPD }\end{array}$ & $\begin{array}{l}\text { TPSSh/def2- } \\
\text { QZVPPD(a) }\end{array}$ & $\begin{array}{l}\text { RPA@TPSSh/ } \\
\text { def2-QZVPPD }\end{array}$ & $\begin{array}{c}\text { 2c-BH-LYP/dhf- } \\
\text { TZVP-2c }\end{array}$ \\
\hline$T_{h}$ & ${ }^{4} A_{u}$ & 0.01 & 0.22 & 0.01 & 0.01 & 0.01 & 0.12 & 0.00 \\
\hline$D_{3 d^{(b)}}$ & ${ }^{4} A_{2 u}$ & 0.01 & 0.24 & 0.00 & 0.16 & 0.00 & 0.13 & 0.01 \\
\hline $\mathrm{C}_{5 \mathrm{v}}(\mathrm{c})$ & ${ }^{4} E_{2}$ & 0.00 & 0.06 & 0.06 & 0.06 & 0.05 & 0.05 & 0.03 \\
\hline
\end{tabular}

(a) $\quad \mathrm{Al}_{\mathrm{h}}\left({ }^{4} \mathrm{~T}_{2 u}\right)$ species lies at $0.20 \mathrm{eV}$ (TPSSh/def2-SVP) and is virtually isoenergetic with respect to the $\mathrm{T}_{h}\left({ }^{4} \mathrm{Au}_{u}\right)$ species at the $2 \mathrm{C}-\mathrm{BH}$ LYP/dhf-TZVP-2c level. The corresponding TPSSh/def2-SVP KS determinant undergoes symmetry breaking to the other species.

(b) $\quad \mathrm{D} \mathrm{D}_{5 \mathrm{~d}}\left({ }^{4} \mathrm{~A}_{2 u}\right)$ species presents symmetry breaking at the TPSSh/def2-SVP level; the broken-symmetry SCF solution corresponds to the $C_{S} / C_{5 v}$ species.

(c) At the TPSSh/def2-SVP level presents slight symmetry breaking, giving a $C_{s}$ nearly $C_{5 v}$ geometry. By all the other computational levels the $C_{5 v}$ optimized structure appears to be slightly lower or nearly equal in energy. 
Table S10: Geometries in cartesian coordinates ( $(\AA)$ for the isomers of tables 1-8 and S1. The computational level is TPSSh/def2-SVP (default) or 2C-TPSSh/dhf-TZVP-2c (see tables 1-4) (the m4 grid has been used).

$\mathrm{Ta}^{-}$

1

$\mathrm{C}_{1} \quad{ }^{2} \mathrm{~A}$

Ta

$\mathrm{Ta}$

$\mathrm{Ta}$

Ta

$\mathrm{Ta}$

$\mathrm{Ta}$

2

$\begin{array}{rrrr}\mathrm{D}_{2 \mathrm{~h}} & { }^{4} \mathrm{Ag} & & \\ & 0.0000000 & 0.0000000 & -1.5745664 \\ 1.5424416 & 1.3411199 & 0.0000000 \\ -1.5424416 & 1.3411199 & 0.0000000 \\ -1.5424416 & -1.3411199 & 0.0000000 \\ 0.0000000 & 0.0000000 & 1.5745664 \\ 1.5424416 & -1.3411199 & 0.0000000\end{array}$

3

$\begin{array}{ccrr}\mathrm{D}_{2 \mathrm{~h}} & { }^{2} \mathrm{~B}_{3 \mathrm{~g}} & & \\ & 0.0000000 & 0.0000000 & -1.5617329 \\ 1.5880019 & 1.3167539 & 0.0000000 \\ -1.5880019 & 1.3167539 & 0.0000000 \\ -1.5880019 & -1.3167539 & 0.0000000 \\ 0.0000000 & 0.0000000 & 1.5617329 \\ 1.5880019 & -1.3167539 & 0.0000000\end{array}$

4

\begin{tabular}{lrrr}
\multicolumn{1}{c}{$\mathrm{C}_{1} / \mathrm{D}_{2 \mathrm{~h}}$} & & & \\
$\mathrm{Ta}$ & 0.0002348 & -1.5571383 & -0.0004479 \\
$\mathrm{Ta}$ & -1.5200445 & -0.0003316 & 1.3329451 \\
$\mathrm{Ta}$ & 1.5202337 & 0.0001514 & 1.3331212 \\
$\mathrm{Ta}$ & 1.5202758 & 0.0002365 & -1.3329870 \\
$\mathrm{Ta}$ & -0.0001357 & 1.5571500 & 0.0003818 \\
$\mathrm{Ta}$ & -1.5205642 & -0.0000680 & -1.3330131
\end{tabular}

5

$\begin{array}{lrrr}C_{1} / C_{2 v} & & & \\ a & 0.0003544 & 0.2162153 & 1.6043715 \\ a & 1.6568991 & 1.2228832 & -0.0011643 \\ a & 1.2910723 & -1.4377338 & 0.0000747 \\ a & -1.2902967 & -1.4381266 & 0.0015731 \\ a & -0.0005607 & 0.2143605 & -1.6046178 \\ a & -1.6574685 & 1.2224014 & -0.0002372\end{array}$

6

$\mathrm{C}_{1}$

$\begin{array}{lll}0.2989687 & 0.2517142 & -1.5604641\end{array}$

Ta

$-1.4607453 \quad 1.4006320$

$-0.2449466$

$\mathrm{Ta}$

$-1.5411876-1.2066285$

0.0310142 
$-1.6299410$

$-0.0837317$

$\mathrm{Ta}_{7}{ }^{-}$

1

$\mathrm{C}_{1}$

${ }^{1} \mathrm{~A}$

$\mathrm{Ta}$

$-2.1169428$

$-0.3141652$

0.3638318

$-1.0246226$

1.8642473

$-0.4775412$

$\mathrm{Ta}$

$-0.0313656$

$-0.2991359$

$-1.5884379$

$\mathrm{Ta}$

0.0373763

0.3314221

1.5828046

Ta

$-0.3258550$

$-2.1317769$

0.1677171

1.4787290

$-0.4448889$

$\mathrm{Ta}$

1.9222987

$-0.9293203$

0.3965145

2

$C_{1}$

${ }^{3} \mathrm{~A}$

3

$C_{3 v}$

$-2.1300902$

$-0.3541398$

0.1337266

$-0.9946582$

1.9515614

$-0.2348267$

$-0.0409007$

$-0.3426661$

$-1.5893095$

0.0433374

0.3645614

1.5664661

$-0.3130463$

$-2.1426364$

0.2849233

1.5000455

1.4310863

$-0.5610608$

1.9353125

$-0.9077668$

0.4000810

4

$\mathrm{C}_{\mathrm{s}}$

$$
{ }^{5} \mathbf{A}_{1}
$$

0.8444613

$1.4626498-0.6360357$

$-0.7565860$

1.3104453

1.4749209

1.5131719

0.0000000

1.4749209

$-0.7565860$

$-1.3104453$

1.4749209

0.8444613

$-1.4626498$

$-0.6360357$

$-1.6889225$

0.0000000

$-0.6360357$

0.0000000

0.0000000

$-2.5166555$

$\mathrm{C}_{1}$

$$
{ }^{3} \mathrm{~A} "
$$

$$
1.3417638
$$

1.0493503

0.0000000

1.2259115

$-1.1523082$

1.3624318

1.2259115

$-1.1523082$

$-1.3624318$

$-0.9750876$

$-1.7737848$

0.0000000

$-1.0149932$

0.3403684

$-1.5057006$

$-1.0149932$

0.3403684

1.5057006

$-0.7885127$

2.3483142

0.0000000

${ }^{1} \mathrm{~A}$ 


$\begin{array}{llrr}\text { Ta } & 1.4233092 & -1.5689780 & -0.0169856 \\ \text { Ta } & 0.9054158 & 0.8034742 & -1.3449594 \\ \text { Ta } & 2.4802800 & 0.6157524 & 0.6571823\end{array}$

6

$\mathrm{C}_{1}$

Ta

$\begin{array}{lll}-0.6727976 & 2.0275649 & -0.2389959\end{array}$

$\mathrm{Ta}$

$\begin{array}{lll}1.7249611 & 1.2845990 & 0.1622830\end{array}$

$\mathrm{Ta}$

$\begin{array}{lll}0.0077105 & -0.0162077 & -1.5924671\end{array}$

$\mathrm{Ta}$

$\begin{array}{lll}0.0147630 & -0.0040722 & 1.5928883\end{array}$

Ta

$\begin{array}{lll}-2.1372980 & 0.0172936 & 0.2328604\end{array}$

$\mathrm{Ta}$

$\begin{array}{lll}1.7512235 & -1.2689164 & -0.0119463\end{array}$

$\mathrm{Ta}$

$\begin{array}{lll}-0.6885625 & -2.0402612 & -0.1446223\end{array}$

7

$$
C_{1} / D_{5 h}
$$

$\mathrm{Ta}$

$\mathrm{Ta}$

$\mathrm{Ta}$

Ta

Ta

$\mathrm{Ta}$

$\mathrm{Ta}$

$\begin{array}{rrr}-1.7529614 & -1.2735409 & 0.0000612 \\ -1.7529690 & 1.2735559 & 0.0000509 \\ 0.0000217 & 0.0000136 & -1.5808068 \\ -0.0001928 & -0.0000138 & 1.5808052 \\ 0.6697163 & -2.0604821 & -0.0000225 \\ 0.6696948 & 2.0604793 & -0.0000376 \\ 2.1666903 & -0.0000120 & -0.0000505\end{array}$

8

$\mathrm{C}_{1} / \mathrm{C}_{3 \mathrm{v}}$

0.8317058

1.4416856

$-0.6262962$

$\begin{array}{ll}-0.7510141 & 1.3005702\end{array}$

1.4606437

1.5025906

$-0.0000391$

1.4600924

$\mathrm{Ta}$

$-0.7503520$

$-1.2994726$

1.4605956

Ta

$0.8315847-1.442776$

$-0.6268850$

Ta

$-1.6655657$

$-0.0006629$

$-0.6274221$

Ta

0.0010507

0.0006952

$-2.5007284$

$\mathrm{Ta}^{-}$

1

$\begin{array}{ccrr}\mathrm{C}_{2 v} & { }^{2} \mathrm{~B}_{1} & & \\ & 0.0000000 & -1.4901400 & 1.8727872 \\ & 0.0000000 & 1.4901400 & 1.8727872 \\ & -1.6687244 & 0.0000000 & 0.6567381 \\ & 0.0000000 & 1.9429318 & -0.6650997 \\ & 1.3112116 & 0.0000000 & -1.8644255 \\ & 1.6687244 & 0.0000000 & 0.6567381 \\ & 0.0000000 & -1.9429318 & -0.6650997 \\ & -1.3112116 & 0.0000000 & -1.8644255 \\ & & & \\ \text { Cs } & { }^{4} \mathbf{A}^{\prime} & & \end{array}$




$\begin{array}{lrrr}\text { Ta } & 1.3720367 & 0.6680563 & 1.3990255 \\ \text { Ta } & -0.6237055 & 1.6678325 & 0.0000000 \\ \text { Ta } & -1.0276484 & -0.1953412 & -2.0371415 \\ \text { Ta } & 2.5946046 & -1.1091139 & 0.0000000 \\ \text { Ta } & 1.3720367 & 0.6680563 & -1.3990255 \\ \text { Ta } & -1.0276484 & -0.1953412 & 2.0371415 \\ \text { Ta } & -2.5796928 & -0.0277165 & 0.0000000 \\ \text { Ta } & -0.0799830 & -1.4764322 & 0.0000000\end{array}$

3

$\mathrm{Ta}$
$\mathrm{Ta}$
$\mathrm{Ta}$
$\mathrm{Ta}$
$\mathrm{Ta}$
$\mathrm{Ta}$
$\mathrm{Ta}$
$\mathrm{Ta}$

6

7

8

$\mathrm{C}_{2 \mathrm{v}}$ $\mathrm{Ta}$ $\mathrm{Ta}$

$C_{3 v}$

Cs
${ }^{2} \mathrm{~A}_{1}$

1.2088194

2.4153721

1.2088194

0.0000000

0.0000000

$-1.2076860$

$-2.4176389$

$-1.2076860$

2.0937367

0.0000000

$-2.0937367$

0.0000000

0.0000000

$-2.0917736$

0.0000000

2.0917736

${ }^{6} \mathrm{~B}_{2}$

$-0.8952607$

0.8952607

1.1135461

$-1.1135461$

1.1135461

$-1.1135461$

$-0.8952607$

0.8952607

$-0.8952607$

0.8952607

1.1135461

$-1.1135461$

$-1.1135461$

1.1135461

0.8952607

$-0.8952607$

$$
{ }^{4} \mathrm{~A}^{4}
$$

2.8415212

1.1432582

1.1432582

$-0.4147045$

0.5231936

$-1.2427670$

$-1.2427670$

$-2.7509926$

$-0.6866127$

0.4358663

0.4358663

1.8994502

$-1.9017863$

$-0.5850811$

$-0.5850811$

0.9873785

$-2.3998335$

$-2.3998335$

0.6851550

0.6851550

$-0.6851550$

$-0.6851550$

2.3998335

2.3998335

0.0000000

1.5128529

$-1.5128529$

0.0000000

0.0000000

1.3940654

$-1.3940654$

0.0000000

${ }^{4} \mathbf{A}_{2}$

$\begin{array}{rrr}1.4770217 & 1.3318690 & 0.4723350 \\ 0.0000000 & 0.0000000 & 2.2130990 \\ 0.0000000 & 0.0000000 & -1.6294704 \\ 1.4770217 & -1.3318690 & 0.4723350 \\ 0.0000000 & -2.4708584 & -1.2364842 \\ -1.4770217 & -1.3318690 & 0.4723350 \\ -1.4770217 & 1.3318690 & 0.4723350 \\ 0.0000000 & 2.4708584 & -1.2364842\end{array}$


4

$\mathrm{C}_{1} / \mathrm{C}_{2 \mathrm{v}}$

\begin{tabular}{llll} 
Ta & -0.0000001 & -1.4573980 & 1.8718093 \\
\hline
\end{tabular}

\begin{tabular}{llll} 
Ta & 0.0000001 & 1.4573980 & 1.8718094 \\
\hline
\end{tabular}

$\begin{array}{llll}\text { Ta } & -1.6477949 & 0.0000000 & 0.6354692\end{array}$

$\begin{array}{llll}\text { Ta } & 0.0000000 & 1.9234214 & -0.6474245\end{array}$

$\begin{array}{llll}\text { Ta } & 1.2902055 & 0.0000001 & -1.8598541\end{array}$

$\begin{array}{llll}\text { Ta } & 1.6477949 & 0.0000000 & 0.6354692\end{array}$

$\begin{array}{llll}\text { Ta } & 0.0000000 & -1.9234214 & -0.6474244\end{array}$

$\begin{array}{llll}\text { Ta } & -1.2902055 & -0.0000001 & -1.8598541\end{array}$

5

$\mathrm{C}_{1} / \mathrm{C}_{s}$

$\begin{array}{lll}1.2784936 & 0.8077708 & 1.3754357\end{array}$

$\begin{array}{lll}-0.8194204 & 1.5614824 & -0.0000018\end{array}$

$\begin{array}{lll}-0.9955171 & -0.2796890 & -2.0232846\end{array}$

Ta

$\begin{array}{lll}2.6830785 & -0.8055485 & 0.0000449\end{array}$

$\begin{array}{lll}1.2785598 & 0.8076671 & -1.3754710\end{array}$

$\begin{array}{lll}-0.9954781 & -0.2797711 & 2.0232791\end{array}$

$\begin{array}{lll}-2.5300495 & -0.3654867 & 0.0000368\end{array}$

$\begin{array}{lll}0.1003332 & -1.4464249 & -0.0000390\end{array}$

Tag

$\mathrm{C}_{2 \mathrm{v}} \quad{ }^{3} \mathrm{~B}_{2}$

Ta

$\begin{array}{lll}0.0000000 & 0.0000000 & 1.3796249\end{array}$

Ta

$0.0000000-1.4111090$

$-1.4508050$

$\mathrm{Ta}$

$\begin{array}{ll}1.3577284 & -2.1599687\end{array}$

0.6725847

Ta

1.3577284

2.1599687

0.6725847

$\mathrm{Ta}$

$0.0000000 \quad 1.4111090$

$-1.4508050$

$\mathrm{Ta}$

$2.1421911 \quad 0.0000000$

$-0.5841769$

$\begin{array}{ll}-2.1421911 & 0.0000000\end{array}$

$-0.5841769$

$\mathrm{Ta}$

$-1.3577284 \quad 2.1599687$

0.6725847

$\mathrm{Ta}$

$-1.3577284 \quad-2.1599687$

0.6725847

2

$\mathrm{C}_{1}$

${ }^{3} \mathrm{~A}$

$-2.19902$

$-1.0695442$

$-0.6891443$

0.8999154

0.1121998

1.6186611

0.9052054

$\mathrm{Ta}$

$-0.4010986$

$-0.7043725$

2.0187272

$\mathrm{Ta}$

1.9972334

$-2.0398998$

$-0.4958624$

$-1.4077235$

0.3930959 
3

4

$C_{1}$

$\mathrm{Ta}$

$\mathrm{Ta}$

Ta

Ta

$\mathrm{Ta}$

$\mathrm{Ta}$

Ta

$\mathrm{Ta}$

Ta

5

\begin{tabular}{|c|c|c|c|}
\hline \multicolumn{4}{|c|}{$C_{1} / C_{2 v}$} \\
\hline Ta & -0.0000003 & -1.3642717 & -0.0000633 \\
\hline $\mathrm{Ta}$ & -0.0000240 & 1.4398139 & -1.3909959 \\
\hline Тa & 1.3365975 & -0.6710117 & -2.1490455 \\
\hline Ta & 1.3366126 & -0.6711220 & 2.1489481 \\
\hline Ta & 0.0000240 & 1.4398869 & 1.3909649 \\
\hline Ta & 2.1136399 & 0.5844194 & 0.0000986 \\
\hline Ta & -2.1136397 & 0.5844195 & 0.0000769 \\
\hline Ta & -1.3366539 & -0.6709896 & 2.1490046 \\
\hline Ta & -1.3365561 & -0.6711446 & -2.1489884 \\
\hline
\end{tabular}

$\begin{array}{lll}-1.4957011 & 0.1047514 & -1.5235044\end{array}$

$\begin{array}{lll}1.2728406 & -0.1766586 & -1.7171682\end{array}$

\section{$C_{s}$}

${ }^{5} \mathrm{~A}$

Ta

$-1.3605766$

0.4982104

$-2.0070688$

$-0.1113529$

1.6573066

0.0000000

2.4517617

0.8406605

0.0000000

1.0538977

$-0.2883040$

$-1.8911941$

$-2.8280476$

0.3107873

0.0000000

$-1.3605766$

0.4982104

2.0070688

$-0.7881921$

$-1.4033262$

0.0000000

1.0538977

$-0.2883040$

1.8911941

1.8891887

$-1.8252412$

0.0000000

${ }^{1} \mathrm{~A}$

0.6361191

$-0.2783674$

$-1.4915771$

1.0727957

1.6295776

0.8347971

$-1.0579227$

1.6293374

$-0.8537043$

2.9303916

0.7062484

$-0.6699047$

$-1.9782498$

$-0.9092887$

$-1.0184761$

$-2.9240292$

0.7403351

0.6609993

$-0.0106135$

$-2.3480029$

0.0140269

$-0.6386436$

$-0.2549526$

1.4947104

1.9701533

$-0.9148870$

1.0291287

$\mathrm{C}_{1} / \mathrm{C}_{\mathrm{s}}$

Ta

$\mathrm{Ta}$

$-1.3593108$

0.4782119

$-1.9898081$

$-0.1104259$

1.6313769

$-0.0000028$

2.4357188

0.8306773

$-0.0000463$

1.0393265

$-0.2790713$

$-1.8644032$

$-2.7940763$

0.3364764

0.0000236

$-1.3593382$

0.4781707

1.9898438

$-0.7745856$

$-1.3883665$

$-0.0000504$

1.0392996

$-0.2790459$

1.8643946

1.8833919

$-1.8084296$

0.0000488 
$\mathrm{Ta}_{10}{ }^{-}$

1

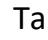

Ta

Ta

Ta

$\mathrm{Ta}$

$\mathrm{Ta}$

Ta

$\mathrm{Ta}$

$\mathrm{Ta}$

Ta

2

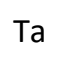

Ta

Ta

$\mathrm{Ta}$

$\mathrm{Ta}$

$\mathrm{Ta}$

$\mathrm{Ta}$

Ta

$\mathrm{Ta}$

$\mathrm{Ta}$

3

Ta

Ta

$\mathrm{Ta}$

$\mathrm{Ta}$

$\mathrm{Ta}$

Ta

$\mathrm{Ta}$

$\mathrm{Ta}$

Ta

Ta

4

Ta

$\mathrm{Ta}$

$\mathrm{Ta}$

$\mathrm{Ta}$

Ta

Ta

$\mathrm{Ta}$

$\mathrm{Ta}$

$\mathrm{Ta}$

Ta
$D_{4 d}{ }^{2} B_{1}$

$\begin{array}{lll}1.8616340 & 0.7711141 & -1.0507656\end{array}$

$0.7711141-1.8616340 \quad-1.0507656$

$0.0000000 \quad 0.0000000 \quad-2.6885209$

$1.8616340-0.7711141 \quad 1.0507656$

$-0.7711141-1.8616340 \quad 1.0507656$

$-0.7711141 \quad 1.8616340 \quad-1.0507656$

$-1.8616340 \quad-0.7711141-1.0507656$

$\begin{array}{lll}0.0000000 & 0.0000000 & 2.6885209\end{array}$

$0.7711141 \quad 1.8616340 \quad 1.0507656$

$\begin{array}{lll}-1.8616340 & 0.7711141 & 1.0507656\end{array}$

$\mathrm{C}_{3 \mathrm{v}} \quad{ }^{4} \mathrm{~A}_{2}$

$0.0000000 \quad 0.0000000-1.5667736$

$0.7850093 \quad 1.3596760 \quad 1.5171436$

$2.4800844 \quad 0.0000000 \quad-0.0774652$

$\begin{array}{lll}-1.2400422 & 2.1478161 & -0.0774652\end{array}$

$\begin{array}{lll}1.2702619 & 2.2001581 & -0.9174205\end{array}$

$0.7850093-1.3596760 \quad 1.5171436$

$-1.2400422-2.1478161 \quad-0.0774652$

$\begin{array}{lll}1.2702619 & -2.2001581 & -0.9174205\end{array}$

$\begin{array}{lll}-1.5700186 & 0.0000000 & 1.5171436\end{array}$

$\begin{array}{lll}-2.5405237 & 0.0000000 & -0.9174205\end{array}$

Cs $\quad{ }^{2} A^{\prime}$

$\begin{array}{lll}-3.3888450 & 0.1284244 & 0.0000000\end{array}$

$-1.6536017-1.1094764-1.3906640$

$0.2594308 \quad 0.6423376 \quad-2.1039302$

$\begin{array}{lll}1.5757889 & 1.6290621 & 0.0000000\end{array}$

$\begin{array}{lll}2.6288034 & -0.4309360 & 1.2851101\end{array}$

$2.6288034-0.4309360 \quad-1.2851101$

$\begin{array}{lll}0.5198637 & -1.4049194 & 0.0000000\end{array}$

$\begin{array}{lll}0.2594308 & 0.6423376 & 2.1039302\end{array}$

$\begin{array}{lll}-1.1760726 & 1.4435824 & 0.0000000\end{array}$

$-1.6536017-1.1094764 \quad 1.3906640$

$C_{s} \quad{ }^{2} A$ '

$\begin{array}{lll}-3.1982867 & 0.5810767 & 0.0000000\end{array}$

$-0.6633895 \quad 1.6096894 \quad 0.0000000$

$\begin{array}{lll}-1.7782560 & 0.0994020 & -1.9965392\end{array}$

$\begin{array}{lll}-1.7782560 & 0.0994020 & 1.9965392\end{array}$

$-1.1147843-1.4907675 \quad 0.0000000$

$0.8926115 \quad-0.1484606 \quad 1.5667224$

$0.8926115-0.1484606-1.5667224$

$\begin{array}{lll}2.0106035 & 1.8529120 & 0.0000000\end{array}$

$\begin{array}{lll}3.2257592 & -0.2653042 & 0.0000000\end{array}$

$\begin{array}{lll}1.5113870 & -2.1894894 & 0.0000000\end{array}$ 
Ta11

1

$\mathrm{C}_{2 \mathrm{v}}$

${ }^{3} \mathbf{B}_{2}$

$\mathrm{Ta}$

$-2.1769725$

$-1.3611203$

0.3378207

$-1.3801122$

0.0000000

$-1.8013750$

$-2.1769725$

1.3611203

0.3378207

0.0000000

2.2004368

$-1.1035590$

0.0000000

2.5173454

1.4955755

0.0000000

0.0000000

1.4674342

2.1769725

1.3611203

0.3378207

0.0000000

$-2.5173454$

1.4955755

1.3801122

0.0000000

$-1.8013750$

0.0000000

$-2.2004368$

$-1.1035590$

2.1769725

$-1.3611203$

0.3378207

2

Cs

$-0.1078907$

${ }^{3} \mathrm{~A} "$

$-2.2075292$

1.2324489

0.7198715

$-1.6335843$

0.1670156

$-1.8265797$

0.0000000

2.1357812

1.3420358

$-2.8389170$

$-1.6461214$

0.2354192

$-1.4684600$

0.0580521

$-1.3943334$

0.0000000

$-2.0170959$

0.9926348

0.0000000

$-0.1078907$

0.7198715

1.6335843

2.1357812

$-1.1831874$

2.2075292

0.1670156

0.2354192

1.4684600

1.3420358

2.8389170

Cs

${ }^{5} A^{\prime}$

$0.0249907 \quad 1.0864381$

0.0000000

1.2725626

$-1.8222579$

0.0000000

2.1947144

0.2142310

$-1.4199768$

0.1211242

1.5030336

$-2.6715554$

$-0.0709619$

$-1.0835270$

$-2.2257117$

2.1947144

0.2142310

1.4199768

$-1.5296026$

$-1.6040344$

0.0000000

$-2.1288521$

0.5361896

$-1.4918300$

0.1211242

1.5030336

2.6715554

$-2.1288521$

0.5361896

1.4918300

$-0.0709619$

$-1.0835270$

2.2257117

4

$\mathrm{C}_{\mathrm{s}}$

3A"

1.0388935

$-1.6346146$

0.0000000

$-1.3188851$

1.2962318

2.1458910

2.7724386

0.2135401

$-1.1975660$

0.6838822

$-0.3753984$

2.5228647

2.7724386

0.2135401

1.1975660

$-1.4542032$

$-1.2545117$

1.3143768 


$\begin{array}{lrrr}\text { Ta } & 0.2526985 & 1.0535282 & 0.0000000 \\ \text { Tа } & -2.6580570 & 0.8213628 & 0.0000000 \\ \text { Ta } & -1.3188851 & 1.2962318 & -2.1458910 \\ \text { Ta } & -1.4542032 & -1.2545117 & -1.3143768 \\ \text { Ta } & 0.6838822 & -0.3753984 & -2.5228647\end{array}$

5

$$
\mathrm{C}_{1}{ }^{\mathbf{3}} \mathbf{A}
$$

$\begin{array}{lll}1.4762074 & 0.8020949 & 1.5834921\end{array}$

$\begin{array}{llll}2.8401847 & 0.4607112 & -0.6569438\end{array}$

$\begin{array}{lll}-0.7063806 & -0.7528629 & 1.5608323\end{array}$

$\begin{array}{lll}-0.7444307 & 2.0996404 & 1.1179284\end{array}$

$\begin{array}{llll}0.9188337 & 2.1480710 & -0.9000495\end{array}$

$\begin{array}{lll}0.6033413 & -0.6327741 & -1.5837910\end{array}$

$\begin{array}{lll}-2.0298906 & -1.4044216 & -0.9300340\end{array}$

$\begin{array}{lll}-2.7838577 & 0.5341955 & 0.6873712\end{array}$

$\begin{array}{lll}-1.4089915 & 1.2118750 & -1.4532516\end{array}$

$\begin{array}{llll}2.0031759 & -1.6539309 & 0.5988400\end{array}$

$-0.1681919 \quad-2.8125976 \quad-0.0243941$

6

$\mathrm{C}_{1}$

${ }^{3} \mathrm{~A}$

$-1.7438037$

$1.4517690 \quad-1.2824434$

$\begin{array}{lll}0.0829165 & 2.8178353 & -0.0143613\end{array}$

$\begin{array}{lll}0.9730755 & 0.6995585 & -1.2901482\end{array}$

$\begin{array}{lll}0.8972789 & -1.2900709 & 1.1450464\end{array}$

$\begin{array}{lll}3.1338610 & -0.1309761 & -0.0863373\end{array}$

$\begin{array}{lll}1.6813091 & 1.3529923 & 1.4297586\end{array}$

$\begin{array}{lll}1.8103436 & -2.0322594 & -1.1880079\end{array}$

$\begin{array}{lll}-0.8354756 & -1.3692259 & -1.0911757\end{array}$

$\begin{array}{lll}-1.8196639 & -1.8744573 & 1.3087970\end{array}$

$\begin{array}{lll}-1.0179535 & 0.7222657 & 1.2574467\end{array}$

$\begin{array}{lll}-3.1618879 & -0.3474313 & -0.1885747\end{array}$

7

$$
\mathrm{C}_{2}
$$

\section{${ }^{5} \mathrm{~A}$}

Ta

$-1.803667$

1.2405185

1.3578301

$\begin{array}{lll}0.0000000 & 0.0000000 & 2.8016733\end{array}$

$\begin{array}{lll}0.8773214 & 1.3810352 & 0.7145854\end{array}$

$\begin{array}{lll}1.0039769 & -1.0008036 & -1.3463691\end{array}$

$\begin{array}{lll}3.1205984 & 0.3144883 & -0.1561301\end{array}$

$\begin{array}{lll}1.8036678 & -1.2405185 & 1.3578301\end{array}$

$\begin{array}{llll}1.6599689 & 1.4337688 & -1.9707529\end{array}$

$\begin{array}{lll}-1.0039769 & 1.0008036 & -1.3463691\end{array}$

$\begin{array}{lll}-1.6599689 & -1.4337688 & -1.9707529\end{array}$

$\begin{array}{lll}-0.8773214 & -1.3810352 & 0.7145854\end{array}$

$\begin{array}{lll}-3.1205984 & -0.3144883 & -0.1561301\end{array}$ 
$\mathrm{Ta}_{12}{ }^{-}$

Cs ${ }^{2} \mathrm{~A} "$

2 $\mathrm{Ta}$

3

3-b
2.2836627

0.1041431

0.6129096

$-1.8176979$

$-2.8949724$

$-0.2292858$

$-1.0118622$

2.2836627

0.1041431

$-1.8176979$

0.6129096

1.7700854

$T_{h}$

0.0000000

0.0000000

0.0000000

$-2.1808312$

$-1.3584441$

$-2.1808312$

$-1.3584441$

2.1808312

1.3584441

0.0000000

2.1808312

1.3584441

$\mathrm{C}_{\mathrm{s}}$

0.2718643

0.1393143

$-1.8861741$

$-2.2815114$

$-1.8861741$

$-0.8293508$

0.6758293

0.6758293

$-0.8293508$

1.7973158

1.7973158

2.3550924

$\mathrm{C}_{\mathrm{s}}$

0.2474854

0.1213814

$-1.8789975$

$-2.2822874$
$-0.8179162 \quad 1.3311450$

$-1.5605445 \quad 2.5399049$

$1.1079358 \quad 2.2572485$

$0.2165845 \quad 2.2068694$

$-0.24580620 .0000000$

$-0.99997140 .0000000$

$1.8136590 \quad 0.0000000$

$-0.8179162-1.3311450$

$-1.5605445-2.5399049$

$0.2165845-2.2068694$

$1.1079358-2.2572485$

$1.5399994 \quad 0.0000000$

$\begin{array}{rr}-2.1808312 & -1.3584441 \\ 2.1808312 & 1.3584441 \\ 2.1808312 & -1.3584441 \\ 1.3584441 & 0.0000000 \\ 0.0000000 & -2.1808312 \\ -1.3584441 & 0.0000000 \\ 0.0000000 & 2.1808312 \\ -1.3584441 & 0.0000000 \\ 0.0000000 & 2.1808312 \\ -2.1808312 & 1.3584441 \\ 1.3584441 & 0.0000000 \\ 0.0000000 & -2.1808312\end{array}$

2A"

$\begin{array}{rr}0.7750048 & 0.0000000 \\ -2.2502258 & 0.0000000 \\ -0.9072947 & -1.3369148 \\ 1.4183854 & 0.0000000 \\ -0.9072947 & 1.3369148 \\ 1.2013045 & -2.5253183 \\ -1.0935565 & -2.3721257 \\ -1.0935565 & 2.3721257 \\ 1.2013045 & 2.5253183 \\ 1.2061622 & -2.1041215 \\ 1.2061622 & 2.1041215 \\ -0.7563954 & 0.0000000\end{array}$

\section{${ }^{4} \mathrm{~A} "$}

$\begin{array}{rr}0.7419310 & 0.0000000 \\ -2.2456203 & 0.0000000 \\ -0.8874425 & -1.3413131 \\ 1.4380006 & 0.0000000\end{array}$




$\begin{array}{lrrr}\text { Ta } & -1.8789975 & -0.8874425 & 1.3413131 \\ \text { Ta } & -0.8169702 & 1.1810782 & -2.5699613 \\ \text { Ta } & 0.6921125 & -1.1046350 & -2.3910690 \\ \text { Ta } & 0.6921125 & -1.1046350 & 2.3910690 \\ \text { Ta } & -0.8169702 & 1.1810782 & 2.5699613 \\ \text { Ta } & 1.7854001 & 1.1957795 & -2.1138344 \\ \text { Ta } & 1.7854001 & 1.1957795 & 2.1138344 \\ \text { Ta } & 2.3503308 & -0.7038717 & 0.0000000\end{array}$

3-c

$\mathrm{C}_{1}$

${ }^{6} \mathrm{~A}$

$\mathrm{Ta}$

0.1369041

0.8195322

$-0.0425057$

0.3142063

$-2.2291875 \quad-0.0621851$

$-1.8642163$

$-1.0366858 \quad-1.2500643$

$-2.4597321$

1.1631446

0.2543196

$-1.7019682$

$-1.1206277$

1.4505483

$-0.9795109$

1.2028204

$-2.4579067$

0.6267204

$-1.0007865$

$-2.4632291$

$0.9051884-1.0310007$

2.3126796

$-0.8230789$

1.1085606

2.5835004

1.7112812

1.3094558

$-2.1749507$

1.7609295

1.3527940

1.9855152

2.3732765

$-0.5380194$

$-0.1357215$

4

$\mathrm{C}_{1}$

${ }^{2} \mathrm{~A}$

$\mathrm{Ta}$

0.7306185

0.1013034

$-0.0699400$

$-2.2536901$

0.1236263

$-0.0191167$

$-1.0015493$

$-1.7361058$

1.5517538

1.1969261

$-2.5412647$

0.0871647

$-1.0350444$

$-1.9980500$

$-1.2144800$

1.3093094

$-0.8818951$

2.3976080

$-0.9279110$

0.9585653

2.1939165

$-1.0013481$

$\begin{array}{ll}0.6207340 & -2.3871585\end{array}$

1.1527496

$-0.9205474$

$-2.4066592$

1.4326782

1.8077147

1.7224812

1.2642742

2.0246483

$-1.6392892$

$-0.8670130$

2.4412710

$-0.2162805$

5

$\mathrm{C}_{1}$

${ }^{4} \mathrm{~A}$

$\mathrm{Ta}$

0.7106051

$-0.0297806$

$-0.0154849$

$-2.2547855$

0.0992367

0.0234972

$-1.0299971$

$-1.7771879$

1.5893624

1.151238

$-2.5904647$

0.0410576

$-1.040775$

$-1.9941895$

$-1.2312554$

1.2505630

$-0.8458350$

2.4427005

$-0.9009341$

0.9509180

2.2267626

$-0.9830363$

$\begin{array}{ll}0.6097447 & -2.3360949\end{array}$

1.2124545

$-0.7722599$

$-2.4842665$

1.3919943

1.9513622

1.5244716 


$\begin{array}{lrrr}\text { Ta } & 1.3289631 & 1.9777245 & -1.5868113 \\ \text { Ta } & -0.8362895 & 2.4207315 & -0.1939389\end{array}$

6

$$
\text { Cs } \quad{ }^{2} \mathrm{~A} \text { ' }
$$

$\begin{array}{lll}1.9054945 & 1.2611795 & 0.0000000\end{array}$

$\begin{array}{lll}-2.3011488 & -1.9136693 & 0.0000000\end{array}$

$\begin{array}{lll}-1.6945363 & -0.4427421 & -2.1484897\end{array}$

$\begin{array}{lll}0.9639263 & 0.1549704 & -2.4742483\end{array}$

$\begin{array}{lll}-0.4782776 & 1.9195143 & 1.2903910\end{array}$

$\begin{array}{lll}-1.6945363 & -0.4427421 & 2.1484897\end{array}$

$\begin{array}{lll}0.9639263 & 0.1549704 & 2.4742483\end{array}$

$\begin{array}{lll}-2.6356896 & 0.7663790 & 0.0000000\end{array}$

$\begin{array}{lll}-0.4782776 & 1.9195143 & -1.2903910\end{array}$

$\begin{array}{lll}0.0961806 & -0.8998351 & 0.0000000\end{array}$

$\begin{array}{lll}2.6764692 & -1.2387696 & -1.1839063\end{array}$

$\begin{array}{lll}2.6764692 & -1.2387696 & 1.1839063\end{array}$

7

$\mathrm{C}_{\mathrm{S}}{ }^{2} \mathrm{~A} "$

$\mathrm{Ta}$

$\begin{array}{lll}-2.2960336 & -0.4743444 & 1.3468837\end{array}$

$\begin{array}{lll}-2.2960336 & -0.4743444 & -1.3468837\end{array}$

$\begin{array}{lll}0.1279988 & -1.0584933 & -2.1921495\end{array}$

$\begin{array}{lll}-0.9671932 & -2.3614804 & 0.0000000\end{array}$

$\begin{array}{lll}0.1279988 & -1.0584933 & 2.1921495\end{array}$

$\begin{array}{lll}1.6066185 & -1.9822947 & 0.0000000\end{array}$

$\begin{array}{lll}1.8059785 & 2.3129868 & 0.0000000\end{array}$

$\begin{array}{lll}-0.1961858 & 1.5247715 & 1.5330948\end{array}$

$\begin{array}{lll}2.3059556 & 0.1553670 & 1.3762516\end{array}$

$\begin{array}{lll}-2.3288737 & 1.7361869 & 0.0000000\end{array}$

$\begin{array}{lll}-0.1961858 & 1.5247715 & -1.5330948\end{array}$

$\begin{array}{lll}2.3059556 & 0.1553670 & -1.3762516\end{array}$

8

$$
\mathrm{C}_{2 \mathrm{v}} \quad{ }^{2} \mathrm{~A}_{\mathbf{1}}
$$

$\begin{array}{lll}-2.1496590 & 1.2590787 & -0.7169022\end{array}$

$\begin{array}{lll}-2.1496590 & -1.2590787 & -0.7169022\end{array}$

$\begin{array}{lll}0.0000000 & 0.0000000 & -2.0505284\end{array}$

$\begin{array}{lll}0.0000000 & -2.6655644 & -0.9370284\end{array}$

$\begin{array}{lll}0.0000000 & 0.0000000 & 0.8323615\end{array}$

$\begin{array}{lll}-1.2273993 & -2.4140511 & 1.4899582\end{array}$

$\begin{array}{lll}2.1496590 & -1.2590787 & -0.7169022\end{array}$

$\begin{array}{lll}1.2273993 & -2.4140511 & 1.4899582\end{array}$

$\begin{array}{lll}1.2273993 & 2.4140511 & 1.4899582\end{array}$

$\begin{array}{lll}2.1496590 & 1.2590787 & -0.7169022\end{array}$

$\begin{array}{lll}0.0000000 & 2.6655644 & -0.9370284\end{array}$

$\begin{array}{lll}-1.2273993 & 2.4140511 & 1.4899582\end{array}$

9

$\mathrm{C}_{2}$

${ }^{2} \mathrm{~A}$

$\begin{array}{lll}1.1896344 & 0.4908584 & -2.3760586\end{array}$

$\mathrm{Ta}$

$\begin{array}{lll}0.6214867 & -1.6375614 & -0.5392184\end{array}$ 


$\begin{array}{lrrr}\mathrm{Ta} & 2.9456930 & -0.7974161 & -0.8405049 \\ \mathrm{Ta} & -1.1896344 & -0.4908584 & -2.3760586 \\ \mathrm{Ta} & -2.0441045 & -1.5002902 & -0.0042584 \\ \mathrm{Ta} & -2.9456930 & 0.7974161 & -0.8405049 \\ \mathrm{Ta} & -2.1436057 & 0.6041641 & 1.6948863 \\ \mathrm{Ta} & 0.3292390 & 1.3333849 & 2.0651540 \\ \mathrm{Ta} & -0.3292390 & -1.3333849 & 2.0651540 \\ \mathrm{Ta} & 2.1436057 & -0.6041641 & 1.6948863 \\ \mathrm{Ta} & -0.6214867 & 1.6375614 & -0.5392184 \\ \mathrm{Ta} & 2.0441045 & 1.5002902 & -0.0042584\end{array}$

10

$$
\mathrm{C}_{\mathrm{S}} \quad{ }^{4} \mathrm{~A}^{\prime}
$$

0.0000000

2.1993873

0.4798899

2.1301515

3.1259392

0.4327729

2.3124178

1.0865119

$-0.7212712$

0.0000000

2.1993873

$-1.7005986-1.4095639$

$-1.2135150$

0.4327729

$-2.3124178$

1.0865119

$-1.1213056$

0.0000000

$-3.2213600$

$-1.7005986$

1.4095639

$-1.7543817$

0.2115397

1.2620407

$-0.5962185$

1.9731165

0.0000000

$-3.2213600$

0.4798899

$-2.1301515$

0.2115397

$-1.2620407$

$$
\mathrm{C}_{\mathrm{i}}
$$

$$
-1.0961843
$$

2.4021983

$\mathrm{C}_{1}$

\section{${ }^{4} \mathbf{A}$}

$-0.9633273-0.0112804$

$-2.3725043$

$\begin{array}{lll}1.6359759 & -1.1973001 & -2.2018002\end{array}$

$\begin{array}{lll}-1.4286643 & 2.4245774 & -0.6277989\end{array}$

$\begin{array}{lll}-0.3988968 & -2.1575597 & -0.8401018\end{array}$

$\begin{array}{lll}3.2040786 & 0.1610243 & -0.5926655\end{array}$

$\begin{array}{llll}0.5851437 & 0.6847577 & -0.3486828\end{array}$

$\begin{array}{lll}1.9823532 & -1.9263570 & 0.4667213\end{array}$

$\begin{array}{lll}-2.1992460 & -0.3684427 & 0.0204908\end{array}$ 


$\begin{array}{lrrr}\text { Ta } & -3.2588270 & 1.8774923 & 0.9038400 \\ \text { Ta } & -0.2999261 & -1.2280460 & 1.7980707 \\ \text { Ta } & 1.9563373 & 0.3183258 & 1.9025609 \\ \text { Ta } & -0.8150023 & 1.4228094 & 1.8918708\end{array}$

13

$$
\mathrm{C}_{2}{ }^{2} \mathrm{~B}
$$

1.3160281

1.7235480

1.7123735

$-1.3160281$

$-1.7235480$

1.7123735

$-1.2195614$

0.8723681

1.7146800

$\begin{array}{ll}1.2195614 & -0.8723681\end{array}$

1.7146800

$-2.3686418$

1.7679730

$-0.4944653$

0.2333830

1.9523075

$-0.6694247$

$-1.3070584$

$-0.0345998$

$-2.1806631$

$-2.7783329$

$-0.7115637$

$-0.0825004$

1.3070584

0.0345998

$-2.1806631$

2.3686418

$-1.7679730$

$-0.4944653$

$-0.2333830$

$-1.9523075$

$-0.6694247$

2.7783329

0.7115637

$-0.0825004$

$\mathrm{Ta}_{13}{ }^{-}$

$c_{s}$ $-0.9422222$

$-2.5372782$

$-0.0535242$

$-0.5151967$

$-0.8797486$

$-0.9422222$

$-0.5151967$

$-2.5372782$

1.6359477

1.6359477

1.4956758

2.6594200

1.4956758

$\mathrm{C}_{2 \mathrm{v}}$

0.0000000

0.0000000

1.4541117

$-1.4541117$

0.0000000

$-2.2477189$

$-2.2474758$

1.4541117

0.0000000
${ }^{1} \mathrm{~A}^{\prime}$

1.8038792

2.2720241

$\begin{array}{ll}-0.0429571 & 1.2974179\end{array}$

$\begin{array}{ll}0.7566474 & 0.0000000\end{array}$

$\begin{array}{ll}-0.8542977 & 2.6519996\end{array}$

$\begin{array}{ll}-1.9480932 & 0.0000000\end{array}$

$\begin{array}{ll}1.8038792 & -2.2720241\end{array}$

$-0.8542977-2.6519996$

$\begin{array}{ll}-0.0429571 & -1.2974179\end{array}$

$\begin{array}{ll}-1.5435402 & -1.2759607\end{array}$

$\begin{array}{ll}-1.5435402 & 1.2759607\end{array}$

$0.9008921 \quad 2.4368254$

$\begin{array}{ll}0.6634932 & 0.0000000\end{array}$

$0.9008921 \quad-2.4368254$

${ }^{1} \mathbf{A}_{1}$

$\begin{array}{ll}-1.6298146 & -2.1373191\end{array}$

$\begin{array}{ll}1.6310755 & 2.1368886\end{array}$

$2.4028081-0.0001412$

$2.4028081-0.0001412$

$1.6298146-2.1373191$

$0.0000000-1.3863749$

$\begin{array}{ll}0.0000000 & 1.3868244\end{array}$

$\begin{array}{ll}-2.4028081 & -0.0001412\end{array}$

$-1.6310755 \quad 2.1368886$ 
3

5

-1.4541117
2.2474758
2.2477189
0.0000000

$-2.4028081$ 0.0000000 0.0000000 0.0000000 $\mathrm{Ta}$
Cs

0.5155585

$-0.5163137$

$-2.1766702$

2.1779236

$-0.5163137$

$-2.2038887$

0.0147270

2.1779236

0.5282446

$-2.2038887$

2.1950832

0.5282446

$-0.5206303$

$\mathrm{C}_{2}$

1.2295189

2.9671365

$-1.1039700$

1.1039700

1.5846865

0.0000000

1.4141115

2.3160020

$-1.5846865$

$-2.9671365$

$-1.2295189$

$-1.4141115$

$-2.3160020$

Cs

$\begin{array}{cr}{ }^{3} \mathrm{~A} " & \\ 2.6594359 & 0.0000000 \\ 1.3011421 & -2.2934770 \\ 1.6198587 & 0.0000000 \\ 0.7886641 & -1.4231370 \\ 1.3011421 & 2.2934770 \\ -0.8066719 & -1.3893671 \\ 0.0414895 & 0.0000000 \\ 0.7886641 & 1.4231370 \\ -1.3225900 & -2.2624098 \\ -0.8066719 & 1.3893671 \\ -1.6503975 & 0.0000000 \\ -1.3225900 & 2.2624098 \\ -2.5914753 & 0.0000000\end{array}$

${ }^{1} \mathrm{~A}$

$-2.0040561 \quad 1.1994643$

$-0.12166710 .4055123$

$-0.79376492 .0958958$

$0.7937649 \quad 2.0958958$

$-1.9354265-1.3315074$

$0.0000000-0.4509381$

$2.2120519-0.1634187$

$0.4812291-1.9804772$

$1.9354265-1.3315074$

0.12166710 .4055123

$2.0040561 \quad 1.1994643$

$-2.2120519-0.1634187$

$-0.4812291-1.9804772$

$$
{ }^{3} A^{\prime}
$$

$\begin{array}{lll}-0.2305202 & -1.3337127 & 2.2900481\end{array}$

$\begin{array}{lll}2.2871743 & -0.6391401 & 1.3562892\end{array}$

$\begin{array}{lll}-1.8851500 & 0.6460958 & 2.2243974\end{array}$

$\begin{array}{lll}-0.0717815 & 0.6561267 & 0.0000000\end{array}$

$\begin{array}{lll}2.2950937 & 1.7142592 & 0.0000000\end{array}$

$\begin{array}{lll}0.7978656 & 1.1849370 & 2.5325023\end{array}$

$\begin{array}{lll}-2.0159542 & -1.2297438 & 0.0000000\end{array}$

$-0.2305202 \quad-1.3337127 \quad-2.2900481$

$0.5830855-2.3340004 \quad 0.0000000$

$\begin{array}{lll}2.2871743 & -0.6391401 & -1.3562892\end{array}$

$\begin{array}{lll}-2.7291828 & 1.4769982 & 0.0000000\end{array}$

$\begin{array}{lll}0.7978656 & 1.1849370 & -2.5325023\end{array}$ 
